\title{
Riesgo de uso indebido de opioides prescritos en pacientes con dolor crónico no oncológico en un hospital del sistema mutual en Chile
}

\section{Risk of misuse of prescribed opioids in patients with chronic non- cancer pain in a workers compensation system hospital in Chile}

\author{
V. Villanueva Catalán ${ }^{1}$, J. C. Vélez González² y A. Castro Lara ${ }^{3}$
}

${ }^{1}$ Escuela de Postgrado, Facultad de Medicina. Universidad de Chile. Santiago, Chile. ${ }^{2}$ Hospital del Trabajador, Asociación Chilena de Seguridad. Santiago, Chile. ${ }^{3}$ Dirección de Investigación, Hospital Clínico Universidad de Chile. Santiago, Chile

\section{RESUMEN}

Introducción: En algunos países el uso indebido de opioides está aumentando considerablemente, sin embargo en Chile no hay datos oficiales. El objetivo de este estudio fue identificar y describir a todos los pacientes en tratamiento por dolor crónico no oncológico (DCNO) con riesgo de uso indebido de opioides del Departamento de Rehabilitación del Hospital del Trabajador, Santiago de Chile, entre el 14 de agosto de 2018 y el 2 de febrero de 2020.

Material y métodos: Se realizó un estudio descriptivo transversal en 120 pacientes usuarios de opioides, con diagnóstico de DCNO. Se les aplicó una encuesta estructurada para caracterizarlos en base a datos demográficos, clínicos, uso indebido de opioides $\left(\mathrm{COMM}^{\circledR}\right)$ y calidad de vida relacionada con salud (SF 36 v.2).

Resultados: Se encontraron 35 pacientes $[29,17 \%)$ con riesgo de uso indebido de opioides. La mediana de edad fue de 50,7 años. El tiempo medio de consumo de opioides fue de 32,4 meses. El tramadol en asociación con paracetamol fue el opioide más utilizado. Se observó un mayor uso indebido, estadísticamente significativo, en los pacientes con diagnóstico de amputación ( $p=0,026)$ y consumo de alcohol $(p=0,003)$. Además, el uso indebido se asoció de manera significativa con una menor puntuación en los dominios de rol físico $(p=0,0299)$, salud general $(p=0,0166)$, vitalidad $(p=0)$, salud mental $(p=0)$ y puntuación global de la escala SF $36(p=0,0003)$.
Villanueva Catalán V, Vélez González JC, Castro Lara A. Riesgo de uso indebido de opioides prescritos en pacientes con dolor crónico no oncológico en un hospital del sistema mutual en Chile. Rev Soc Esp Dolor. 2021;28(2):82-91

\section{ABSTRACT}

Introduction: In some countries, the abuse of opioids is increasing considerably, however, in Chile there is no official data. The aim of this study was to identify and to describe all patients in treatment for chronic non-cancer pain (CNCP) at risk of opioid misuse within the Rehabilitation Department of Hospital del Trabajador, Santiago de Chile, from August 14, 2018 to February 02, 2020.

Material and methods: A descriptive cross-sectional study was carried out in 120 opioid users with a diagnosis of CNCP. A structured survey was applied to characterize them based on demographic, clinical data, opioid misuse $\left(\mathrm{COMM}^{\circledR}\right.$ ), and health-related quality of life (SF 36 v.2).

Results: We found 35 patients (29.17\%) with risk of opioid abuse. The median age was 50.7 years. The mean time of opioid consumption was 32.4 months. Tramadol in association with paracetamol was the most widely used opioid. Statistically significant increased misuse was observed in patients diagnosed with amputation ( $p$-value $=0.026$ ) and alcohol use ( $p$-value $=0.003$ ). Furthermore, misuse was significantly associated with a lower score in the domains of physical role (p-value $=0.0299$ ), general health ( $p$-value $=0.0166$ ), vitality ( $p$-value $=0$ ), mental health ( $p$-value $=0$ ) and global score of the SF 36 scale (p-value $=0.0003$ )

Conclusion: Our study showed a 29,1\% prevalence of risk of opioid misuse, which is similar to that found in the literature. There is a relationship between opioid misuse

Recibido: 19-09-2020

Aceptado: 21-03-2021

Correspondencia: Valeria Villanueva Catalán valevillanuevac@gmail.com 
Conclusión: Nuestro estudio arrojó una prevalencia de un 29,1\% de riesgo de uso indebido de opioides, siendo esta similar a la encontrada en la literatura. Existe una relación entre uso indebido de opioides y consumo de alcohol, diagnóstico de amputación y una menor calidad de vida, lo que genera una mayor discapacidad en estos pacientes. Este es el primer informe en Chile al respecto.

Palabras clave: Opioide, dolor crónico, trastornos relacionados con opioides, uso indebido de opioides, dolor crónico no oncológico. and alcohol consumption, amputation diagnosis, and a lower quality of life, which generates greater disability in these patients. This is the first report in Chile.

Key words: Opioid, chronic pain, opioid-related disorders, misuse opioid, chronic non-cancer pain.

\section{INTRODUCCIÓN}

A pesar de la atención internacional por mejorar el manejo del dolor, el tratamiento inadecuado de este se ha convertido en un grave problema de salud pública grave $[1,2]$. Si bien existen varios tratamientos farmacológicos y no farmacológicos, los opioides han ganado cada vez más aceptación como agentes clave para tratar el dolor crónico no oncológico (DCNO) (3-5). Su efecto ha sido demostrado en disminuir el dolor y mejorar la función física de manera significativa en este tipo de pacientes [6]. Sin embargo, su administración a largo plazo puede estar asociada con un mayor riesgo de abuso y adicción [7].

El mal uso y la adicción a los opioides recetados es un problema grave de salud pública en los EE. UU.; el número de muertos por sobredosis casi se ha cuadruplicado en el último siglo $(8,9)$. El uso indebido, mal uso o uso inapropiado se define como el uso de cualquier sustancia de una manera distinta a la indicada o prescrita. El mal uso de opioides representa un riesgo aumentado de presentar comportamientos aberrantes como el abuso (mal uso que conlleva consecuencias negativas para la saludo y/o uso ilegal) y la adicción (trastorno neurobiológico crónico) (10). Un metanálisis reciente calculó una proporción promedio de uso indebido en pacientes con DCNO entre 21 y $29 \%$ (11).

El deterioro de la calidad de vida de los pacientes con diagnóstico de DCNO y mal uso de opioides es un fenómeno analizado y se ha establecido que son las dimensiones relacionadas a la salud física y mental, las más afectadas (12).

Actualmente, en EE. UU. se realiza un esfuerzo significativo por revertir estos efectos negativos de los opioides, incluida una nueva directriz de los Centers for Disease Control and Prevention (CDC) titulada "Prescripción de Opioides para el Dolor Crónico" [8]. Los médicos se encuentran en la difícil posición de proporcionar un alivio adecuado del dolor a la vez que minimizan el uso inapropiado de analgésicos (13).

En Chile, el más reciente "Estudio Nacional de Drogas en Población General" del Servicio Nacional para la Prevención y Rehabilitación del Consumo de Drogas y Alcohol (SENDA), fue publicado en el año 2018.
En este, el consumo de analgésicos opioides sin prescripción médica alcanzaba el 1,2 \% de la población (14). Si bien estos datos son alentadores para Chile, el comportamiento adictivo sigue siendo un problema mundial y más datos sobre posibles pacientes en riesgo en nuestro país podrían ayudar a mantener bajas las tasas de abuso.

Así, como objetivo principal decidimos documentar la prevalencia del riesgo de uso indebido de opioides en pacientes con DCNO secundario a trauma producto de accidentes de trayecto o trabajo del Departamento de Rehabilitación de un Hospital de referencia perteneciente a un sistema mutual. De manera secundaria, describir sus características demográficas, clínicas y calidad de vida.

\section{MATERIALES Y MÉTODOS}

Se realizó un estudio descriptivo transversal en pacientes con diagnóstico de DCNO, de acuerdo con el criterio médico, atendidos de manera ambulatoria en el Departamento de Rehabilitación entre el 14 de agosto de 2018 y el 2 de febrero de 2020 en el Hospital del Trabajador en Santiago (HT), Chile. Se incluyó a pacientes con diagnóstico de DCNO en tratamiento con opioides por un periodo mayor o igual a un mes, mayores de 18 años sin límite de edad, sin distinción de sexo, capaces de leer y hablar en español. Se excluyeron aquellos participantes con diagnóstico de daño orgánico cerebral y en tratamiento por trastorno de adicciones.

Este estudio fue revisado y aprobado por el Comité de Ética de Investigación Científica del HT. Todos los participantes del estudio dieron su consentimiento informado por escrito.

Los 120 pacientes elegibles aceptaron participar en el estudio (100\%). A cada paciente reclutado se le realizó una entrevista estructurada de manera presencial por un miembro del personal de investigación. El objetivo de la entrevista fue obtener características demográficas, antecedentes médicos y hábitos del paciente. Posteriormente, respondían dos cuestionarios autoaplicados donde podían asesorarse con el personal de investigación en caso de dudas: la Encuesta de Cali- 
dad de Vida Relacionada a la Salud (CVRS) SF 36 v. 2 y Current Opioid Misuse Measure (COMM ${ }^{\circledR}$ ].

Por su parte, el personal de investigación revisó la ficha médica electrónica de los participantes del estudio para obtener información detallada de su características clínicas. Todos los datos obtenidos se introdujeron en una base de datos de Microsoft Excel.

Las variables demográficas que se incluyeron fueron: sexo, edad, nivel más alto de educación, estado civil, región de residencia y situación laboral. Las variables clínicas evaluadas fueron el tipo de opioide utilizado, el tiempo de uso, la dosis diaria, la dosis de rescate y el diagnóstico. Para objetivar el nivel de dolor se utilizó la Escala Visual Análoga (EVA) de 11 puntos $[0$ a 10, ambos inclusive). Se evaluó la presencia de comorbilidades médicas, psiquiátricas, antecedentes de abuso de alguna sustancia por el paciente o su familia, consumo de bebidas cafeinadas, energéticas, alcohólicas, tabaquismo actual y pasado.

Para describir la dosis total de medicamentos opioides, se agrupó a los participantes según los miligramos equivalentes de tramadol (15) (la receta de opioide más común], dada la gran cantidad de diferentes tipos de recetas.

Respecto a las encuestas aplicadas, la de CVRS SF 36 v. 2, fue diseñada por Ware y cols. a principios de los noventa y modificada en el año 2000. Este instrumento genérico consta de 36 preguntas y proporciona un perfil de estado de salud. Las dimensiones evaluadas son funcionamiento físico, limitaciones de rol por problema físico, dolor corporal, salud general, vitalidad, funcionamiento social, limitaciones de rol por problema emocional y salud mental del entrevistado. Para cada dimensión, los ítems son codificados, agregados y transformados en una escala con un rango de $\mathrm{O}$ (el peor estado de salud) a 100 (el mejor estado de salud). Se encuentra validado en varios idiomas, incluido el español chileno desde el año $2004(16,17)$.

El COMM ${ }^{\circledast}$ fue desarrollado con el asesoramiento de un grupo de expertos en dolor y adicciones. Su desarrollo y validación inicial fue publicada el 2007 por la International Association for the Study of Pain (IASP) [18]. El cuestionario consta de 17 preguntas auto aplicadas en alrededor de 10 minutos. Las preguntas se puntúan de $\mathrm{O}$ (nunca) a 4 (muy a menudo) según frecuencia de ocurrencia. La puntuación total varía de $\mathrm{O}$ a 68, y una puntuación $\geq 9$ indica un alto riesgo de uso indebido de opioides. El 2011 se realizó una traducción y validación lingüística al español.

El análisis de datos se realizó mediante el uso del programa estadístico Stata v.14. Los resultados se obtuvieron mediante estadística descriptiva, en medidas de tendencia central (promedio, mediana) y medidas de dispersión (desviación estándar, rango, percentiles) para las variables de tipo cuantitativa. Para las variables cualitativas se agruparon en números absolutos y su frecuencia relativa en porcentaje. Para la comparación entre los grupos de variables cuantitativas se aplicó la prueba rangos de Wilcoxon. En el caso de la comparación de 2 o más grupos se aplicó la Prueba de Kruskal Wallis con comparaciones múltiples. En el caso de las variables cualitativas, se aplicó la Prueba de Fisher. Se consideró significativo un p-valor < 0,05.

\section{RESULTADOS}

Ciento veinte participantes cumplieron con los criterios de inclusión y se incluyeron al estudio. La mediana de la edad de los sujetos fue de 50,7 años ( $\pm \mathrm{DE} 12,73$ ). Destaca un porcentaje mayor de hombres en comparación a las mujeres $(72,5$ y $27,5 \%$ y una mayoría laboralmente inactiva (83,3 \%) (Tabla I).

TABLA I

CARACTERÍSTICAS DEMOGRÁFICAS DE LA MUESTRA. LOS VALORES SE PRESENTAN COMO EL PROMEDIO (DE) O EL NÚMERO [\%]

\begin{tabular}{|c|c|}
\hline & $N .^{\circ}[\%]$ \\
\hline \multicolumn{2}{|l|}{ Sexo } \\
\hline Hombre & $87(72,5)$ \\
\hline Mujer & $33(27,5)$ \\
\hline Edad en años (DE) & $50,7( \pm 12,7)$ \\
\hline \multicolumn{2}{|l|}{ Nivel educacional } \\
\hline Básica incompleta & $7(5,8)$ \\
\hline Básica completa & $16(13,3)$ \\
\hline Media incompleta & $23(19,1)$ \\
\hline Media completa & $43(35,8)$ \\
\hline Universitaria o técnica incompleta & $14(11,7)$ \\
\hline Universitaria o técnica completa & $17(14,1)$ \\
\hline \multicolumn{2}{|l|}{ Estado civil } \\
\hline Casado & $56(46,7)$ \\
\hline Divorciado & $8(6,7)$ \\
\hline Separado & $9(7,5)$ \\
\hline Viudo & $6(5,0)$ \\
\hline Soltero con pareja & $20(16,7)$ \\
\hline Soltero sin pareja & $21(17,5)$ \\
\hline \multicolumn{2}{|l|}{ Región de residencia } \\
\hline Coquimbo & $1(0,8)$ \\
\hline Valparaíso & $6(5,0)$ \\
\hline O'Higgins & $1(0,8)$ \\
\hline Maule & $4(3,3)$ \\
\hline Bio-bío & $4(3,3)$ \\
\hline Los Lagos & $2(1,7)$ \\
\hline Los Ríos & $1(0,8)$ \\
\hline Metropolitana & $101(84,1)$ \\
\hline \multicolumn{2}{|l|}{ Situación laboral } \\
\hline Activo & $20(16,7)$ \\
\hline Inactivo & $100(83,3)$ \\
\hline
\end{tabular}


El tiempo promedio de uso de opioides fue de 32 meses, con un rango de 1 hasta 500,6 meses de uso [41 años]. La media de la dosis total diaria en $\mathrm{mg}$ de tramadol fue de 142,2 mg $(37,5-850 \mathrm{mg}$ ), la media de dosis de rescate diario en $\mathrm{mg}$ de tramadol fue de $56,5 \mathrm{mg}$ (20-200 mg). El dolor promedio según EVA fue de 4,8 puntos $( \pm$ DE 2,1) (Tabla II).

Destaca el antecedente de consumo de alcohol, tabaquismo actual y tabaquismo en el pasado en un 39,1, 32,5 y $35 \%$, respectivamente (Tabla III).

La puntuación $\mathrm{COMM}^{\circledR}$ promedio obtenido por la muestra fue de 6,5 puntos (0-28 puntos). El 29,17\%

TABLA II

CARACTERÍSTICAS CLIINICAS DE LA MUESTRA. LOS VALORES SE PRESENTAN COMO EL PROMEDIO (DE) O EL NÚMERO (\%)

\begin{tabular}{|c|c|}
\hline & N. ${ }^{\circ}[\%]$ \\
\hline \multicolumn{2}{|l|}{ Tipo de opioide } \\
\hline Tramadol & $40(33,3)$ \\
\hline Tramadol/paracetamol & $94(78,3)$ \\
\hline Buprenorfina & $3(2,5)$ \\
\hline Tapentadol & $5(4,2)$ \\
\hline Meses de uso promedio (DE) & $32,4( \pm 62,7)$ \\
\hline \multicolumn{2}{|l|}{ Diagnóstico } \\
\hline Amputación & $12(10,0)$ \\
\hline Fractura & $73(60,8)$ \\
\hline Lesión medular & $4(3,3)$ \\
\hline Raquioestenosis & $1(0,8)$ \\
\hline Hombro doloroso & $9(7,5)$ \\
\hline Infección partes blandas & $12(10,0)$ \\
\hline Dolor lumbar & $6(5,0)$ \\
\hline Gonalgia & $1(0,8)$ \\
\hline Coxalgia & $1(0,8)$ \\
\hline Traumatismo encéfalo craneano & $2(1,7)$ \\
\hline Lesión nervio periférico & $9(7,5)$ \\
\hline Politraumatismo & $9(7,5)$ \\
\hline $\begin{array}{l}\text { Sindrome de dolor regional } \\
\text { completo }\end{array}$ & $3(2,5)$ \\
\hline $\begin{array}{l}\text { Dosis diaria en mg de tramadol } \\
\text { [DE] }\end{array}$ & $\begin{array}{c}142,2 \\
( \pm 114,0) \\
\end{array}$ \\
\hline $\begin{array}{l}\text { Dosis sos diaria en mg de } \\
\text { tramadol (DE) }\end{array}$ & $56,5[ \pm 37,2]$ \\
\hline Dolor promedio según EVA (DE) & $4,8( \pm 2,2)$ \\
\hline \multicolumn{2}{|l|}{ Comorbilidades } \\
\hline Hipertensión arterial & $32(26,7)$ \\
\hline Diabetes mellitus & $20(16,7)$ \\
\hline Dislipidemia & $3(2,5)$ \\
\hline Sobrepeso u obesidad & $23(19,1)$ \\
\hline
\end{tabular}

de los sujetos presentaban un uso indebido de opioides. La puntuación total de la encuesta SF 36 fue de 44,13 ( \pm DE 15,0) (Tabla IV).

\section{TABLA III}

ANTECEDENTES MÓRBIDOS DE LA MUESTRA

\begin{tabular}{|l|c|}
\hline \multicolumn{2}{|l|}{$N^{\circ}[\%]$} \\
\hline Ant. familiar adicción & $1(0,8)$ \\
\hline Ant. personal adicción & $1(0,8)$ \\
\hline $\begin{array}{l}\text { Ant. personal enfermedad } \\
\text { psiquiátrica }\end{array}$ & $23(19,2)$ \\
\hline Consumo de bebidas cafeinadas \\
\hline Nunca & $4(3,3)$ \\
\hline Menos de una vez a la semana & $8(6,7)$ \\
\hline Una vez o más a la semana & $72(60,0)$ \\
\hline Una vez al día o más & $36(30,0)$ \\
\hline Consumo de bebidas energéticas \\
\hline Nunca & $112(93,3)$ \\
\hline Menos de una vez a la semana & $6(5,0)$ \\
\hline Una vez o más a la semana & $2(1,7)$ \\
\hline Consumo de alcohol & $47(39,1)$ \\
\hline Frecuencia de consumo de alcohol \\
\hline $1-4$ días a la semana & $1(0,8)$ \\
\hline $5-6$ días a la semana & $1(0,8)$ \\
\hline Menos de una vez al mes & $45(37,5)$ \\
\hline Nunca & $73(60,8)$ \\
\hline Tabaquismo actual & $39(32,5)$ \\
\hline Tabaquismo pasado & $43(35)$ \\
\hline
\end{tabular}

TABLA IV

PUNTUACIONES CUESTIONARIOS. LOS VALORES SE PRESENTAN COMO EL PROMEDIO (DE) O EL NÚMERO [\%]

\begin{tabular}{|l|c|}
\hline & $N .{ }^{\circ}[\%]$ \\
\hline \multicolumn{2}{|l|}{ Promedio dimensión SF 36 (DE) } \\
\hline Función física & $55,4[ \pm 26,0]$ \\
\hline Rol físico & $27,2[ \pm 25,3]$ \\
\hline Dolor corporal & $38,9[ \pm 16,5]$ \\
\hline Salud general & $38,8[ \pm 17,9]$ \\
\hline Vitalidad & $48,2[ \pm 18,7]$ \\
\hline Rol social & $43,9[ \pm 18,1]$ \\
\hline Rol emocional & $37,9[ \pm 24,5]$ \\
\hline Salud mental & $63,0[ \pm 21,0]$ \\
\hline Puntuación total SF 36 (DE) & $44,13[ \pm 15,0]$ \\
\hline Puntuación COMM ${ }^{\circledR}$ promedio (DE) & $6,5[ \pm 6,0]$ \\
\hline Puntuación COMM ${ }^{\circledR} \geq \mathbf{9}$ (\%) & $35(29,2]$ \\
\hline
\end{tabular}


El 26,4 \% de los hombres presentó un uso indebido de opioides, mientras que en las mujeres este valor alcanzó el 36,4 \% ( $p=0,198$ ). Los pacientes laboralmente activos presentaron una prevalencia similar a los inactivos, con un 25 y $30 \%$, respectivamente $(p=0,439)$. La prevalencia de uso inadecuado más alta se obtuvo en los niveles educacionales menores (básica incompleta y básica completa) con un 42,9 y 43,8 \%, respectivamente. Los pacientes separados presentaron el porcentaje más alto de mal uso de opioides con un $55 \%$ ( $p=0,154$ ) (Tabla V).

Tramadol presentó una mayor prevalencia de uso indebido con $37,5 \%(p=0,114)$, seguido de buprenorfina con 33,3 \% ( $p=0,648)$. Los diagnósticos con mayor prevalencia de uso indebido fueron coxalgia (100\%) ( $p=0,292)$, síndrome de dolor regional complejo [66,7\%) ( $p=0,203$ ) y amputación [58,3 \%) ( $p=0,026$ ]. La comorbilidad más asociada a mal uso de opioides fue el sobrepeso u obesidad [34,8 \%] ( $p=0,337)$, seguida de la dislipidemia [33,3\%) ( $p=0,648$ ). La hipertensión arterial presenta la prevalencia más alta de uso adecuado de opioides con un $84,4 \%$ ( $p=0,037$ ) (Tabla VI).
Los pacientes con antecedentes de consumo de alcohol presentaron los niveles más altos de consumo indebido de opioides con un 44,7\% ( $p=0,003$ ) (Tabla VII).

En la puntuación total de SF 36 se observó un promedio menor en los pacientes que presentaban mal uso de opioides respecto a quienes presentaban un uso adecuado ( $p=0,0003$ ) (Tabla VIII).

No se encontraron asociaciones estadísticamente significativas en las características demográficas de los grupos con uso adecuado $\left(\mathrm{COMM}^{\circledR}<9\right.$ ) e indebido $\left[\mathrm{COMM}^{\circledR} \geq 9\right.$ ) de opioides (Tabla V).

Se observó una diferencia estadísticamente significativa en la asociación de uso indebido de opioides y el diagnóstico de amputación ( $p=0,026$ ) y el uso adecuado de opioides con el antecedente de hipertensión arterial ( $p=0,037$ ) (Tabla VI).

Hubo una asociación estadísticamente significativa en la asociación de uso indebido de opioides y consumo de alcohol ( $p=0,003$ ) (Tabla VII).

Se observó una diferencia estadísticamente significativa en la asociación de uso indebido de opioides y puntuación total de la evaluación SF 36 ( $p=0,0003$ ) y en sus dimensiones: rol físico ( $p=0,0299)$, salud general $(p=0,0166)$, vitalidad $(p=0,000)$ y salud mental $(p=0,000)$ (Tabla VIII)

TABLA V

PUNTUACIÓN COMM ${ }^{\odot}$ SEGÚN CARACTERÍSTICAS DEMOGRÁFICAS. CARACTERÍSTICAS DEMOGRÁFICAS DE LA MUESTRA. LOS VALORES SE PRESENTAN COMO EL PROMEDIO (DE) O EL NÚMERO (\%)

\begin{tabular}{|c|c|c|c|}
\hline & $\begin{array}{c}\text { Uso adecuado } \\
N^{\circ}{ }^{\circ}(\%)\end{array}$ & $\begin{array}{c}\text { Uso indebido } \\
N^{\circ}[\%]\end{array}$ & $\mathrm{p}$ value \\
\hline \multicolumn{3}{|l|}{ Sexo } & \multirow{3}{*}{0,198} \\
\hline Hombre & $64(73,6)$ & $23(26,4)$ & \\
\hline Mujer & $21(63,6)$ & $12[36,4)$ & \\
\hline Edad en años (DE) & $50,0( \pm 13,0)$ & $48,6[ \pm 13,4]$ & 0,4488 \\
\hline \multicolumn{3}{|l|}{ Nivel educacional } & \multirow{7}{*}{0,564} \\
\hline Básica incompleta & $4(57,1)$ & $3(42,9)$ & \\
\hline Básica completa & $9(56,2)$ & $7(43,8)$ & \\
\hline Media incompleta & $18(78,3)$ & $5(21,7)$ & \\
\hline Media completa & $32(74,4)$ & $11[25,6]$ & \\
\hline Universitaria o técnica incompleta & $11(78,6)$ & $3(21,6)$ & \\
\hline Universitaria o técnica completa & $11(64,7)$ & $6(35,3)$ & \\
\hline \multicolumn{3}{|l|}{ Estado civil } & \multirow{7}{*}{0,154} \\
\hline Casado & $37(66,1)$ & $19(33,9)$ & \\
\hline Divorciado & $5(62,5)$ & $3(37,5)$ & \\
\hline Separado & $4(44,4)$ & $5(55,6)$ & \\
\hline Viudo & $6(100)$ & $0(0)$ & \\
\hline Soltero con pareja & $17(81,0)$ & $4(19,0)$ & \\
\hline Soltero sin pareja & $16(80,0)$ & $4(20,0)$ & \\
\hline \multicolumn{3}{|l|}{ Situación laboral } & \multirow{3}{*}{0,439} \\
\hline Activo & $15(75,0)$ & $5(25,0)$ & \\
\hline Inactivo & $70(70,0)$ & $30(30,0)$ & \\
\hline
\end{tabular}


TABLA VI

PUNTUACIÓN COMM ${ }^{\circ}$ SEGÚN CARACTERÍSTICAS CLÍNICAS.

LOS VALORES SE PRESENTAN COMO EL PROMEDIO (DE) O EL NÚMERO (\%)

\begin{tabular}{|c|c|c|c|}
\hline & $\begin{array}{c}\text { Uso adecuado } \\
N .^{\circ}[\%]\end{array}$ & $\begin{array}{c}\text { Uso indebido } \\
N .{ }^{\circ}[\%]\end{array}$ & $\mathrm{p}$ value \\
\hline \multicolumn{4}{|l|}{ Tipo de opioide } \\
\hline Tramadol & $25(62,5)$ & $15(37,5)$ & 0,114 \\
\hline Tramadol/paracetamol & $70(74,5)$ & $24(25,5)$ & 0,08 \\
\hline Buprenorfina & $2(66,7)$ & $1[33,3]$ & 0,648 \\
\hline Tapentadol & $4(80,0)$ & $1(20,0)$ & 0,544 \\
\hline Meses de uso promedio (DE) & $27( \pm 49,0)$ & $36,3( \pm 82,0)$ & 0,1039 \\
\hline \multicolumn{4}{|l|}{ Diagnóstico } \\
\hline Amputación & $5(41,7)$ & $7[58,3]$ & 0,026 \\
\hline Fractura & $53(72,6)$ & $20(27,4)$ & 0,37 \\
\hline Lesión medular & $3(75,0)$ & $1(25,0)$ & 0,667 \\
\hline Raquioestenosis & $1(100)$ & $0(0)$ & 0,708 \\
\hline Hombro doloroso & $7(77,8)$ & $2(22,2)$ & 0,481 \\
\hline Infección partes blandas & $9(75,0)$ & $3(25,0)$ & 0,516 \\
\hline Dolor lumbar & $5(83,3)$ & $1(16,7)$ & 0,434 \\
\hline Gonalgia & $1(100)$ & $0(0)$ & 0,708 \\
\hline Coxalgia & $\mathrm{O}(\mathrm{O})$ & $1(100)$ & 0,292 \\
\hline Traumatismo encéfalo craneano & $1(50)$ & $1(50)$ & 0,5 \\
\hline Lesión nervio periférico & $6(66,7)$ & $3(33,3)$ & 0,519 \\
\hline Politraumatismo & $7[77,8)$ & $2(22,2)$ & 0,481 \\
\hline Síndrome de dolor regional completo & $1(33,3)$ & $2(66,7)$ & 0,203 \\
\hline Dosis diaria en $\mathrm{mg}$ de tramadol (DE) & $141,0[ \pm 121,8]$ & $137,7( \pm 84,5)$ & 0,514 \\
\hline Dolor promedio según EVA (DE) & $4,9( \pm 2,0)$ & $5,2[ \pm 2,4]$ & 0,4537 \\
\hline \multicolumn{4}{|l|}{ Comorbilidades } \\
\hline Hipertensión arterial & $27(84,4)$ & $5(15,6)$ & 0,037 \\
\hline Diabetes mellitus & $16(80)$ & $4(20)$ & 0,241 \\
\hline Dislipidemia & $2(66,7)$ & $1[33,3]$ & 0,648 \\
\hline Sobrepeso u obesidad & $15(65,2)$ & $8(34,8)$ & 0,337 \\
\hline
\end{tabular}

\section{DISCUSIÓN}

La prevalencia del riesgo de uso indebido de opioides en tratamiento por DCNO en nuestro estudio fue de un $29,1 \%$, lo cual es concordante con lo publicado en la literatura (19-22). En el año 2017, Voon y Karamouzian (23) publicaron un metanálisis de 38 estudios, en el cual la prevalencia del mal uso de opioides se encontraba en un rango entre 21 al $29 \%$.

Estudios previos indican que las personas que son más jóvenes, solteros, desempleados y con un nivel educativo más bajo, tienen más probabilidades de abusar o depender de los medicamentos opioides recetados (24-26). Esto se condice con la prevalencia de uso inadecuado más alta encontrada en nuestro estudio en los niveles educacionales menores (básica incompleta y básica completa). Los pacientes solteros no representaron el mayor uso indebido de opioides, sí los pacientes separados con un $55 \%$ ( $p=0,154)$. Con respecto a la inactividad laboral, esta solo fue levemente mayor en los pacientes con uso indebido respecto al uso adecuado, con un 30 y un $25 \%$, respectivamente. La edad no obtuvo una diferencia estadísticamente significativa en ambos grupos en nuestra muestra.

La evidencia actual informa que la hidrocodona y la oxicodona (de liberación prolongada como inmediata) son las drogas de elección en el $75 \%$ de los pacientes con abuso de opioides [27]. No se identificaron pacientes usuarios de oxicodona en nuestra muestra, ya que no era parte del arsenal farmacológico del HT al momento del estudio. El tramadol fue el opioide más prescrito y con mayor uso indebido en nuestra investigación. 
TABLA VII

PUNTUACIÓN COMM ${ }^{\odot}$ SEGÚN ANTECEDENTES MÓRBIDOS

\begin{tabular}{|c|c|c|c|}
\hline & $\begin{array}{c}\text { Uso adecuado } \\
\text { N. }{ }^{\circ}[\%]\end{array}$ & $\begin{array}{c}\text { Uso indebido } \\
N .^{\circ}[\%]\end{array}$ & $\mathrm{p}$ value \\
\hline Ant. familiar adicción & $1(100)$ & $0(0)$ & 0,708 \\
\hline Ant. personal adicción & $1(100)$ & $0[0]$ & 0,708 \\
\hline Ant. personal enfermedad psiquiátrica & $14(60,9)$ & $9(39,1)$ & 0,179 \\
\hline \multicolumn{3}{|l|}{ Consumo de bebidas cafeinadas } & \multirow{5}{*}{0,332} \\
\hline Nunca & $3(75,0)$ & $1(25,0)$ & \\
\hline Menos de una vez a la semana & $5(62,5)$ & $3[37,5)$ & \\
\hline Una vez o más a la semana & $55(76,4)$ & $17[23,6)$ & \\
\hline Una vez al día o más & $22(61,1)$ & $14(38,9)$ & \\
\hline \multicolumn{3}{|l|}{ Consumo de bebidas energéticas } & \multirow{4}{*}{0,319} \\
\hline Nunca & $81(72,3)$ & $31(27,7)$ & \\
\hline Menos de una vez a la semana & $3(50,0)$ & $3(50,0)$ & \\
\hline Una vez o más a la semana & $1[50]$ & $1(50,0)$ & \\
\hline Consumo de alcohol & $26(55,3)$ & $21(44,7)$ & 0,003 \\
\hline \multicolumn{4}{|l|}{ Frecuencia de consumo de alcohol } \\
\hline 1-4 días a la semana & $1(100)$ & $0(0)$ & 0,699 \\
\hline 5-6 días a la semana & $0(0)$ & $1(100)$ & \\
\hline Menos de una vez al mes & $25(55,6)$ & $20(44,4)$ & \\
\hline Tabaquismo actual & $29(74,4)$ & $10(25,6)$ & 0,357 \\
\hline Tabaquismo pasado & 43 [35] & $11(26,2)$ & 0,379 \\
\hline
\end{tabular}

TABLA VIII

PUNTUACIÓN COMM ${ }^{\odot}$ SEGÚN SF 36 V.2.

\begin{tabular}{|c|c|c|c|}
\hline & Uso adecuado media $N{ }^{\circ}[\%]$ & Uso indebido media N. ${ }^{\circ}[\%]$ & $\mathrm{p}$ value \\
\hline \multicolumn{4}{|l|}{ Promedio dimensión SF 36} \\
\hline Función física & $58,0( \pm 25,5)$ & $48,6( \pm 26,2)$ & 0,0836 \\
\hline Rol físico & $29,8( \pm 25,0)$ & $20,9( \pm 25,3)$ & 0,0299 \\
\hline Dolor corporal & $40,4[ \pm 16,6]$ & $35,2( \pm 15,7)$ & 0,0885 \\
\hline Salud general & $41,0( \pm 17,5)$ & $33,5( \pm 18,1)$ & 0,0166 \\
\hline Vitalidad & 53,3 ( $\pm 16,8]$ & $35,7[ \pm 17,4]$ & 0 \\
\hline Función social & $46,1( \pm 18,6)$ & $38,7( \pm 16,2]$ & 0,0515 \\
\hline Rol emocional & $40,5( \pm 24,4)$ & $31,7( \pm 23,9)$ & 0,0547 \\
\hline Salud mental & $68,6( \pm 20,1)$ & $49,4( \pm 16,3)$ & 0 \\
\hline Puntuación total SF 36 & $47,2( \pm 14,0)$ & $37,8( \pm 14,8)$ & 0,003 \\
\hline
\end{tabular}

Este resultado es similar al informado por Zhang y cols., quienes describieron que el largo periodo de uso $\mathrm{y} / \mathrm{o}$ las altas dosis de tramadol pueden ser un importante factor de riesgo de uso indebido, incluso para aquellos sin abuso previo de drogas (28).

Con respecto al tiempo de uso y su asociación con el uso indebido, existe literatura que respalda la asociación entre el mayor tiempo de uso y el mal uso [29].
En nuestra muestra, si bien no existe una asociación estadísticamente significativa, el tiempo de uso promedio es mayor en los pacientes con uso indebido comparado con el uso adecuado, con 27 y 36,3 meses respectivamente.

Está descrito que el uso indebido de opioides tendría relación con algunos diagnósticos como el DCNO post accidente automovilístico [30] y la cervicalgia [31]. 
En nuestra muestra resultó una asociación estadísticamente significativa con el diagnóstico de amputación. Creemos que en nuestro estudio la patología de salud mental fue subdiagnosticada y en este caso pudiese estar actuando como factor contribuyente a esta asociación. Es sabido que el uso indebido tiene una fuerte asociación con la ansiedad, depresión y el trastorno por estrés postraumático, entre otros $[29,30]$. Aun así, los números son pequeños y sería interesante si nuestros hallazgos se pudiesen reproducir en muestras más grandes, por ejemplo, mediante análisis secundario de una gran base de datos.

Los pacientes clasificados en otros estudios con alto riesgo de abuso de opioides informan más dolor subjetivo, múltiples quejas de dolor y un mayor grado de limitaciones relacionadas con el dolor [30). En nuestra muestra las puntuaciones de dolor según EVA son muy similares para ambos grupos. También está descrita la asociación de uso indebido y mayor dosis de opioide [29); sin embargo, en nuestra investigación las dosis fueron similares para ambos grupos.

Los números para "antecedente personal de adicción" y "antecedente familiar de adicción" fueron muy pequeños $(n<5)$ en nuestro estudio, lo que explica resultados no significativos para estos factores de riesgo bien descritos [32). Esto pudiese estar en contexto de una respuesta socialmente esperada y sería interesante en investigaciones futuras indagar de manera más objetiva estos antecedentes.

Por otro lado, en nuestro estudio se encontró una asociación entre el consumo de alcohol y el uso indebido de opioides estadísticamente significativa $(p=0,003)$. Esta asociación se encuentra ampliamente descrita en la literatura $(29,33,34)$. Si bien la metodología no nos permite hacer una declaración sobre causalidad, la alta prevalencia del consumo de alcohol debe tenerse en cuenta al tratar a pacientes con abuso de opioides. El consumo de tabaco se también encuentra ampliamente descrito como factor asociado al mal uso de opioides (29), sin embargo, en nuestra muestra no se observó relación significativa.

Cuando se analizó la calidad de vida de los pacientes incluidos en el estudio, se observaron diferencias estadísticamente significativas entre ambos grupos de uso adecuado e indebido y la puntuación global de la SF 36. El porcentaje ponderado global de todos los pacientes fue de 44,13 ( $\pm D E 15,0$ ), lo que es significativamente más bajo que la puntuación promedio en la población chilena, el cual es 76,1 (16). Estudios previos describen la asociación entre el mal uso de opioides y un peor funcionamiento físico y mental (12). En nuestro estudio destaca una diferencia significativa para cuatro dominios de la SF 36 (limitación del rol físico, salud general, vitalidad y salud mental), lo que nos parece concordante con estos hallazgos.

Es imperativa la creación de guías clínicas basadas en la evidencia científica, destinadas a mejorar la comunicación entre los médicos y pacientes sobre los riesgos y beneficios de la terapia con opioides para el dolor crónico, mejorar su seguridad y la eficacia, y reducir los riesgos asociados al tratamiento con opioides a largo plazo.

\section{Fortalezas y Limitaciones}

La presente investigación tiene como fortaleza ser la primera en Chile que estudia una población de pacientes de una mutualidad manejados con opioides para el tratamiento del DCNO en busca de un uso indebido. Además, también es el primer estudio en Chile en utilizar como el $\mathrm{COMM}^{\circledR}$ como herramienta de riesgo para uso problemático de consumo de opioides. Su relevancia clínica radica en las asociaciones que surgen entre algunas características clínicas y el uso indebido de opioides, las cuales deben ser consideradas al momento de prescribirlos.

La principal limitación del estudio es que solo se incluyeron pacientes de un centro hospitalario privado, por lo que no se puede afirmar que la muestra es representativa del país. Aun así, da una primera pista sobre qué proporción del uso indebido de opioides en pacientes con diagnóstico de DCNO se puede encontrar a nivel nacional.

Se utilizó la validación lingüística al español del $\mathrm{COMM}^{\circledast}$, sin embargo, esta versión no se encuentra validada en la población chilena. Además, el $\mathrm{COMM}^{\circledast}$ parece identificar a algunos pacientes como positivos, resultando en falsos positivos: pacientes identificados con un uso indebido de opioides cuando no lo son [18]. Es necesario tener precaución al interpretar las puntuaciones $\mathrm{COMM}^{\circledR}$ y tener en cuenta otras circunstancias atenuantes [35]. Por ejemplo, es posible que algunos sujetos en este estudio hubiesen demostrado comportamientos consistentes con la adicción debido al dolor controlado de manera inadecuada.

Por otra parte, en nuestro estudio no se evaluaron variables correspondientes a otros factores que predisponen a que los pacientes con DCNO presenten una alta prevalencia de uso indebido de opioides, como el uso concomitante con cannabis [36), benzodiacepinas y antidepresivos [29].

Por último, el diseño transversal del estudio no permite que los pacientes sean seguidos en el tiempo, lo que limita las inferencias posibles. A pesar de esto, el tamaño de la muestra fue lo suficientemente grande como para arrojar resultados inequívocos e incluso estadísticamente significativos.

Nuestros resultados respaldan la necesidad de realizar estudios multicéntricos prospectivos, en los cuales el $\mathrm{COMM}^{\circledast}$ se administre de manera repetida, además de realizar un diagnóstico más objetivo de la patología de salud mental.

\section{CONCLUSIÓN}

Nuestro estudio mostró que el 29,1\% de los pacientes en tratamiento con opioides con diagnóstico de DCNO presentan un riesgo elevado de mal uso de estos fármacos. Esta prevalencia es similar a la encontrada en la literatura.

El consumo de alcohol debe ser examinado y tratado en los pacientes con DCNO, y la alta coincidencia de mal uso y consumo del alcohol debe reconocerse al hacerlo. Asimismo, es importante tener en consideración la alta prevalencia de uso indebido de opioides en los pacientes con diagnóstico de amputación traumática. 


\section{CONFLICTO DE INTERESES}

Los autores declaran que no tienen ningún conflicto de intereses.

\section{BIBLIOGRAFÍA}

1. Joranson D, Ryan KM, Aaron M, Dahl JL. Trends in medical use and abuse of opioid analgesics. JAMA. 2000;28(13)3:17104. DOI: 10.1001/jama.283.13.1710.

2. Gilson AM, Ryan KM, Joranson DE, Dahl JL. A reassessment of trends in the medical use and abuse of opioid analgesics and implications for diversion control: 1997-2002. J Pain Sym Manage. 2004;28(2):176-88. DOI: 10.1016/j.jpainsymman.2004.01.003.

3. American Pain Society. Principles of analgesic use in the treatment of acute pain and cancer pain. 4th ed. Glenview, IL: American Pain Society; 1999.

4. American Pain Society. Guideline for the management of pain in osteoarthritis, rheumatoid arthritis, and juvenile chronic arthritis. Clinical practice guideline number 2. Glenview, IL: American Pain Society. 2002.

5. Michna E, Ross EL, Hynes WL, Nedeljkovic SS, Soumekh S, Janfaza $D$, et al. Predicting aberrant drug behavior in patients treated for chronic pain: importance of abuse history. J Pain Sym Manage. 2004;28(3):250-8. DOI: 10.1016/j.jpainsymman.2004.04.007.

6. Busse JW, Wang L, Kamaleldin M, Craigie S, Riva JJ, Montoya $L$, et al. Opioids for Chronic Noncancer Pain: A Systematic Review and Meta-analysis. JAMA. 2018;320(23):2448-60. DOI: 10.1001/jama.2018.18472.

7. National Institutes of Health $(\mathrm{NIH})$. Prescription opioid use and abuse in the treatment of pain [Internet]. RFA-DA-O6-O05 [Accessed on 1/18/2007]. Available at: http://grants.nih. gov/grants/guide/rfa-files/RFA-DA-O6-O05.html.

8. Frieden TR, Houry D. Reducing the Risks of Relief The CDC Opioid- Prescribing Guideline. N Engl J Med. 2016;374(16):1501-4. DOI: 10.1056/NEJMp1515917.

9. National Institute on Drug Abuse: Overdose Death Rates [Internet]. [cited 2017 Jan 11]. Available from: https:// www. drugabuse.gov/related-topics/trends-statistics/overdose-death-rates.

10. Vidal J. Manual de medicina del dolor. Fundamentos, evaluación y tratamiento. Madrid: Sociedad Española del Dolor; 2016. p. 132.

11. Vowles KE, McEntee ML, Julnes PS, Frohe T, Ney JP, van der Goes DN. Rates of opioid misuse, abuse, and addiction in chronic pain: a systematic review and data synthesis. Pain. 2015;156(4):569-76. DOI: 10.1097/01.j.pa in.0000460357.01998.f1.

12. Ashrafioun L, Bohnert A, Jannausch M, Ilgen M. Evaluation of the Current Opioid Misuse Measure Among Substance Use Disorder Treatment Patients. J Subst Abuse Treat. 2015;55(2015):15-20. DOI: 10.1016/j. jsat.2015.02.007.

13. Hampton T. Physicians advised on how to offer pain relief while preventing opioid abuse. JAMA. 2004;292(10):11646. DOI: 10.1001/jama.292.10.1164.

14. Ministerio del Interior y Seguridad Pública Gobierno de Chile. SENDA. Décimo teRcer estudio nacional de drogas en población general [Internet]. Senda; 2018. Disponible en: https:// www. senda.gob.cl/wp-content/uploads/2020/02/ ENPEG-2018.pdf.
15. Torres-Morera LM. Tapentadol retard en el dolor crónico intenso. Rev Soc Esp Dolor. 2011;18(5):283-90.

16. Olivares-Tirado P. Estado de salud de beneficiarios del sistema de salud de Chile: 2004-2005: documento de trabajo. Departamento de Estudios y Desarrollo. [Internet] Superintendencia de Isapres; 2006. Disponible en: https://www. supersalud. gob.cl/documentacion/666/articles-1062 recurso 1.pdf.

17. Aróstegui I, Núñez-Antón V. Aspectos estadísticos del cuestionario de calidad de vida relacionada con salud Short Form36 (SF-36). Estadística Española 2008;50(167):147-92.

18. Butler SF, Budman SH, Fernandez KC, Houle B, Benoit C, Katz $\mathrm{N}$, et al. Development and validation of the Current Opioid Misuse Measure. Pain. 2007;130(1-2):144-56. DOI: 10.1016/j.pain.2007.01.014.

19. Chou R, Turner JA, Devine EB, Hansen R, Sullivan S, Blazina I, et al. The effectiveness and risks of long-term opioid therapy for chronic pain: a systematic review for a National Institutes of Health Pathways to Prevention Workshop. Ann Intern Med. 2015;162(4):276-86. DOI: 10.7326/M14-2559.

20. Noble M, Tregear SJ, Treadwell JR, Schoelles K. Longterm opioid therapy for chronic noncancer pain: a systematic review and meta-analysis of efficacy and safety. J Pain Symptom Manag. 2008;35(2):214-28. DOI: 10.1016/j. jpainsymman.2007.03.015.

21. Martell BA, O'Connor PG, Kerns RD, Becker W, Morales $\mathrm{K}$, Kosten T, et al. Systematic review: opioid treatment for chronic back pain: prevalence, efficacy, and association with addiction. Ann Intern Med. 2007;146(2):116-27. DOI: 10.7326/0003-4819-146-2-200701160-00006.

22. Fishbain DA, Cole B, Lewis J, Rosomoff HL, Rosomoff RS. What percentage of chronic nonmalignant pain patients exposed to chronic opioid analgesic therapy develop abuse/addiction and/or aberrant drug-related behaviors? A structured evidence-based review. Pain Med. 2008;9(4):444-59. DOl: 10.1111/j.1526-4637.2007.00370.x.

23. Voon P, Karamouzian M, Kerr T. Chronic pain and opioid misuse: a review of reviews. Subst Abuse Treat Prev Policy. 2017;12(1):36. DOI: 10.1186/s13011-017-0120-7.

24. Becker WC, Sullivan LE, Tetrault JM, Desai RA, Fiellin DA. Non-medical use, abuse and dependence on prescription opioids among US adults: psychiatric, medical and substance use correlates. Drug Alcohol Depend. 2008:94(1-3):38-47. DOI: 10.1016/j.drugalcdep.2007.09.018.

25. Becker WC, Fiellin DA, Desai RA. Nonmedical use, abuse and dependence on sedatives and tranquilizers among US adults: psychiatric and socio-demographic correlates. Drug Alcohol Depend. 2007:90(2-3):280-7. DOI: 10.1016/j. drugalcdep.2007.04.009.

26. Becker WC, Fiellin DA, Merrill JD, Schulman B, Finkelstein $R$, Olsen $Y$, et al. Opioid use disorder in the United States: insurance status and treatment access. Drug Alcohol Depend. 2008:94(1-3):207-13. DOl: 10.1016/j.drugalcdep.2007.11.018

27. Cicero TJ, Ellis MS, Paradis A, Ortbal Z. Determinants of fentanyl and other potent $\mu$ opioid agonist misuse in opioid- dependent individuals. Pharmacoepidemiol Drug Saf. 2010:19(10): 1057-63. DOl: 10.1002/pds. 1989.

28. Zhang H, Liu Z. The investigation of tramadol dependence with no history of substance abuse: a cross-sectional survey of spontaneously reported cases in Guangzhou City, China. Biomed Res Int. 2013;2013:283425. DOI: $10.1155 / 2013 / 283425$.

29. Honorato D.C, Kimiko R. Opioid dependence in chronic pain patients. Rev Dor. São Paulo. 2011;12(2):160-5. 
30. Kaye AD, Jones MR, Kaye AM, Ripoll J, Galan V, Beakley B, et al. Prescription Opioid Abuse in Chronic Pain: An Updated Review of Opioid Abuse Predictors and Strategies to Curb Opioid Abuse: Part 1. Pain Physician. 2017;20(2S):S93S109. DOI: 10.36076/ppj.2017.s109.

31. Just JM, Bingener L, Bleckwenn M, Schnakenberg R, Weckbecker $\mathrm{K}$. Risk of opioid misuse in chronic non-cancer pain in primary care patients - a cross sectional study. BMC Fam Pract. 2018;19(1):92. DOI: 10.1186/s12875-018-0775-9.

32. Turk DC, Swanson KS, Gatchel RJ. Predicting opioid misuse by chronic pain patients: a systematic review and literature synthesis. Clinl J Pain. 2008;24(6):497-508. DOI: 10.1097/AJP.Ob013e31816b1070.

33. Meltzer EC, Rybin D, Saitz R, Samet JH, Schwartz SL, Butler $\mathrm{SF}$, et al. Identifying prescription opioid use disorder in pri- mary care: diagnostic characteristics of the Current Opioid Misuse Measure (COMM). Pain. 2011;152(2):397-402. DOI: 10.1016/j.pain.2010.11.006.

34. Butler SF, Budman SH, Fanciullo GJ, Jamison RN. Cross validation of the current opioid misuse measure to monitor chronic pain patients on opioid therapy. Clin J Pain. 2010;26(9):7706. DOI: 10.1097/AJP.Ob013e3181f195ba.

35. Savage SR. Assessment for addiction in pain-treatment settings. Clin J Pain. 2002;18(4 Suppl.):S28-38. DOI: 10.1097/00002508-200207001-00004.

36. Reisfield GM, Wasan AD, Jamison RN. The prevalence and significance of cannabis use in patients prescribed chronic opioid therapy: a review of the extant literature. Pain Med. 2009;10(8):1434-41. DOI: 10.1111/j.15264637.2009.00726.x. 